\title{
Educación virtual en la satisfacción escolar en estudiantes de una institución educativa secundaria de Ayacucho, 2021
}

\author{
Nicanor Piter Saavedra Carrión \\ nicanor.saavedra@ayacucho.edu.pe \\ https://orcid.org/0000-0001-5827-9524 \\ Gabriela Soledad Vásquez Urbina \\ gvasquezur@ucvvirtual.edu.pe \\ https://orcid.org/0000-0002-1286-344X \\ Mónica Patricia Vásquez Urbina \\ mpvasquez20@hotmail.com \\ https://orcid.org/0000-0001-8258-2371 \\ Cindy Vílchez Ramírez \\ vilchez.cindy@gmail.com \\ https://orcid.org/0000-0001-7988-8580 \\ Evelyn Ucharima Huarcaya \\ evelyn0001uh@gmail.com \\ https://orcid.org/0000-0003-0150-0616
}

\section{RESUMEN}

El presente estudio estableció la influencia de la educación virtual en la satisfacción escolar, de tipo aplicado, de nivel explicativo, de enfoque cuantitativo, de método hipotético-deductivo y de nivel no-experimental, correlacional causal. Respecto a la población la conformó un total de 157 estudiantes de una institución educativa de Ayacucho, y por muestreo estratificado se determinó la muestra integrada por 112 estudiantes. La técnica fue la encuesta y como instrumento se seleccionaron dos cuestionarios, validados por expertos y de 0,947 y 0,971 de confiabilidad respectivamente. Los resultados obtenidos por intermedio de la prueba de regresión logística ordinal, evidenciaron que el informe de ajuste de modelo la sig=0,000<0,05, afirmando que el modelo propuesto de educación virtual explica a la satisfacción escolar, y según el Pseudo R2 de Cox y Snell dicho modelo explica en un 24.2\% y según Nagelkerke un 30,5\%, en tal sentido, se concluyó que la educación virtual influye de forma significativa en la satisfacción escolar

Palabras clave: educación virtual; satisfacción escolar; aprendizaje. 


\title{
Virtual education in the school satisfaction in students of a secondary educational institution in Ayacucho, 2021
}

\begin{abstract}
The present study established the influence of virtual education on school satisfaction, of applied type, explanatory level, quantitative approach, hypothetical-deductive method and non-experimental, causal correlational level. The population consisted of a total of 157 students from an educational institution in Ayacucho, and a sample of 112 students was determined by stratified sampling. The technique was the survey and two questionnaires were selected as instruments, validated by experts and with a reliability of 0.947 and 0.971 respectively. The results obtained through the ordinal logistic regression test, evidenced that the model fit report the sig $=0.000<0.05$, affirming that the proposed model of virtual education explains school satisfaction, and according to the Pseudo R2 of Cox and Snell said model explains $24.2 \%$ and according to Nagelkerke $30.5 \%$, in such sense, it was concluded that virtual education has a significant influence on school satisfaction.
\end{abstract}

Keyword: virtual education;school satisfaction; learning.

Artículo recibido: 05 octubre. 2021 Aceptado para publicación: 02 noviembre 2021 Correspondencia: nicanor.saavedra@ayacucho.edu.pe Conflictos de Interés: Ninguna que declarar 


\section{INTRODUCCIÓN}

La emergencia sanitaria a causa del Covid-19, ha generado que se disponga aislamiento social en diversos países del mundo, provocando que los sistemas educativos tomen como medida para salvaguardar la salud de los docentes como de los estudiantes el cierre de las escuelas, perjudicando a más de 1,600 millones de escolares (Naciones Unidas, 2020), ello, ha ocasionado que el profesorado cambie su manera de enseñar, percibiendo variaciones muy significativas sobre su práctica docente, adecuándola a una educación virtual, el cual es considerada como una estrategia de educación a distancia, que impacta sobre la calidad educativa por poseer un carácter multimedia e interactivo (Crisol-Moya et ál., 2020).

A nivel mundial, países como China, de manera inmediata atendieron dicho problema, facilitando dispositivos móviles a los escolares de bajos recursos, con planes de internet, con el objetivo de cerrar la brecha digital y de conexión, En Argentina, se dio el lanzamiento del portal "Seguimos Educando", que ofrece a los escolares de sus tres niveles de educación básica, material educativo y una biblioteca virtual donde se puede hallar libros clásicos digitalizados, y por señal abierta de TV contenido educativo para inicial y primaria (Ministerio de Educación de Argentina, 2020). Asimismo, Colombia, implementó una educación virtual, para toda escuela pública del país la cual es un $85 \%$ de todo el su sistema educativo, y para los escolares de zonas rurales, se facilitó el alcance de material educativo (UNESCO, 2020).

En el ámbito nacional, el MINEDU (2020), dispuso la implementación de la estrategia de educación a distancia “Aprendo en Casa", dando inicio al año escolar 2020 a partir del 6 de abril, y que actualmente durante el año 2021, sigue siendo una alternativa para seguir brindando el servicio educativa, dicha estrategia consiste en la difusión de contenido educacional mediante señal abierta de TV, Radio nacional y por la web, cuyo fin es dar continuidad a la educación y que éste llegue a la mayor cantidad de hogares peruanos. Cabe señalar, que, durante las primeras semanas de su instauración, se han percibido varias problemáticas, siendo las más destacables, la falta de conexión y de dispositivos móviles o digitales, en mayor cantidad de zonas rurales, también la inexperiencia sobre el uso de recursos digitales de gran parte de docentes ha generado insatisfacción sobre la forma cómo se estaba llevando a cabo las acciones de enseñanza.

En la provincia de Fajardo, Ayacucho, es notable la diferencia sobre la participación de 
los escolares en la estrategia "Aprendo en Casa", lo cual es evidenciado en los reportes mensuales elaborados por el profesorado, resultando muy preocupante, ya que ello genera que las metas institucionales se vean afectadas. Finalmente, en una institución educativa de nivel secundario del distrito de Huancapi, se tiene acceso al internet con baja intensidad, asimismo, ocasionalmente la señal de TV es interrumpida por los cambios climáticos del lugar, la señal de radio nacional es repetida por la radio local, pero de igual manera en algunas ocasiones falla, por ello, todo lo descrito repercute en la satisfacción escolar, ocasionando descontento con la atención docente.

Por lo descrito, se ha planteado el siguiente problema: ¿Cómo influye la educación virtual en la satisfacción escolar en estudiantes de una institución educativa secundaria de Ayacucho, 2021?, Asimismo, se planteó como objetivo: Determinar la influencia de la educación virtual en la satisfacción escolar en estudiantes de una institución educativa secundaria de Ayacucho, 2021, y como hipótesis: La educación virtual influye significativamente en la satisfacción escolar en estudiantes de una institución educativa secundaria de Ayacucho, 2021.

Respecto a las bases teóricas, como sustento a la educación virtual, se ha considerado a la teoría Gestalt, la cual propone el fomento de un pensamiento productivo, que se adquiere al ofrecer una comprensión estructural, mediante la percepción significativa y organización de conexiones (Cáceres y Munévar, 2016), además, mencionar que dicha teoría se basa en lo que se llega a percibir y como lo percibido provoca efecto sobre el aprendizaje, en tal sentido, los materiales virtuales, que son utilizados para una educación virtual deben cumplir con principios de percepción "contraste figura-fondo, la sencillez, la proximidad, la similaridad, la simetría y el cierre".

Otra de las teorías es el constructivismo, entendida como la teoría que faculta a la persona por medio de su actividad (física y mental), construir su propio conocimiento, relacionando el conocimiento previo con lo nuevo, estableciendo de esa manera relaciones entre elementos, dando sentido a lo recibido (Vega-Lugo et ál., 2019), es decir, que el aprendizaje se da por medio de la participación activa de la persona, el cual mediante su accionar, logra determinar su respuesta a estimulantes ambientales, en tal sentido, el docente se convierte en orientador, asimismo, relacionado con la educación virtual, la propuesta de entornos virtuales puede orientarse a varios principios de ésta teoría, algunos relacionados al rol protagonista del estudiante relacionado a la 
construcción de su conocimiento, el cual le permite solucionar problemas en el ámbito donde éste se desenvuelve.

Respecto a lo anterior, el modelo educacional "Flip Learning", promueve la reflexión al poseer como generador de calidad al propio educando, buscando la generación de cambios en el paradigma educativo, basado en principios del constructivismo, el cual promueve el trabajo en equipo, planteando el logro de retos individuales y comunes, cuyo resultado es un aprendizaje creativo (Peche y Giraldo, 2019).

Por otro lado, la educación virtual es comprendida como el manejo apropiado de recursos y herramientas tecnológicas para la enseñanza virtual, que equipara la posibilidad de dar oportunidad a todos los estudiantes con ubicación geográfica diferida (Martínez et ál., 2019), asimismo, es una nueva modalidad de enseñanza a distancia diferida de la presencial, que necesita de una formación y gestión de competencias idóneas para autorregular el aprendizaje (Gros, 2018), implica también el uso de variadas herramientas y aplicaciones digitales con el objetivo de facilitar la difusión de la información. Ahmadi y Nourabadi (2020), afirmaron que la educación virtual es el producto del uso de las TIC, que impulsa a la sociedad hacia grandes cambios en el sector educativo, clave para la transición de enfoques tradicionalistas a innovadores.

Respecto a las dimensiones de educación virtual, se consideró la propuesta de Gros (2011), quien identificó cuatro, siendo la primera de las dimensiones los recursos, comprendido con el uso de materiales educativos, cuyo fin es permitir que el estudiante adquiera un aprendizaje significativo, en su contexto el cual el se desenvuelve, es la utilización de medios virtuales de aprendizaje, considerados como espacios educativos que contienen actividades y herramientas, utilizadas provechosamente por el docente y estudiante con interfaz amigable. La segunda dimensión es el acompañamiento, rol que asume el docente adoptando un papel de guía y orientados, innovando su actuación durante el proceso de enseñanza, ayudando a dirigir la obtención de metas preestablecidas. La tercera dimensión es la colaboración, basado en el otorgamiento de protagonismo al proceso comunicativo y al trabajo en equipo de los estudiantes, es decir, se trata de proponer situaciones donde los estudiantes coordinen actuaciones en grupo, gestionando recursos e información. Y la cuarta dimensión es la competencia digital del estudiante, traducido como la percepción de la actuación del escolar, al verse en situaciones donde deba utilizar entornos virtuales eficientemente, es decir, es la 
implicancia de uso creativo, critico y seguro de las TIC, con el objetivo de lograr aspectos relacionados con su desempeño escolar, es la adquisición de habilidades tecnológicas que le faciliten el logro del aprendizaje autónomo.

En relación al sustento de la satisfacción escolar, se ha considerado la teoría de la autodeterminación, la cual postula que las características sociales propias de los sujetos están sometidas a experimentar el bienestar y la vitalidad (Tomás y Gutiérrez, 2019), esta teoría llega a demostrar lo relevante de satisfacer tres necesidades básicas: "autonomía, competencia y relación", con el objetivo de lograr su crecimiento psicológico, bienestar y otros efectos buenos sobre el aprendizaje. Cabe mencionar, que esta teoría argumenta que una de sus variables es la ayuda de la autonomía por "Figuras de Autoridad" la cual facilita el desenvolvimiento de la facilidad.

Respecto a la satisfacción escolar, se entiende como el grado de congruencia de lo que se espera con lo que se obtiene, en relación a la experiencia de concebir una educación a distancia (Zambrano, 2016 y González-Peiteado et ál., 2017), es decir, es el estándar percibido por el estudiante sobre el cumplimiento de sus expectativas, metas o competencias, referidas a su propio aprendizaje, además, hace referencia al nivel de congruencia de los mismos, luego de experimentar una educación en modalidad virtual. También es definida como la medición de la calidad al adquirir un servicio, es según Manrique y Sánchez (2018), el "hablar de su apreciación sobre el servicio que se les brinda" (p. 18).

Sobre las dimensiones, Zambrano (2016), identificó a cinco dimensiones, siendo la primera la competencia digital docente, basado en la actuación creativa, critica y segura de herramientas tecnológicas, con el objetivo de lograr las metas propuestas en relación a la práctica docente, empleabilidad, aprendizaje, participación social, entre otros, asimismo, Esteve et ál. (2018), señaló que un docente es digitalmente competente al poseer una mezcla de "creencias, conocimientos, capacidades y actitudes básicas de la identidad docente" (p. 107). La segunda dimensión es la calidad del curso virtual, basado en la percepción de la propuesta organizada y horario flexible, es la valoración del curso virtual, comparada con la actividad presencial, en tal sentido, De Luca (2020), señaló que el aula virtual es "un espacio o entorno creado virtualmente con la intencionalidad de que un estudiante obtenga experiencias de aprendizaje a través de recursos/materiales formativos bajo la supervisión e interacción con un profesor" (p. 3). La tercera dimensión 
son los recursos tecnológicos, los cuales son parte de una maneta sistémica de diseñar, conducir y evaluar el accionar docente por medio de la utilización de variados recursos que enriquecen la labor docente (Morán et ál., 2017), es decir, que dichos recursos pueden ser de tipo informático, audiovisual y de comunicación, siendo ventajoso para el profesorado, ya que podrá hacer uso durante la clase. La cuarta dimensión es la estructura del medio virtual, basada en la percepción de cómo está el aula virtual, sus aspectos que están incluidos, su utilidad y fácil uso de su sistema, asimismo De Luca (2020), mencionó que el medio virtual no tiene un solo modelo o regla de seguir, sino que la estructura del medio virtual, depende básicamente de varias particularidades, como la necesidad del área curricular, el conocimiento, manejo apropiado de recursos y actividades que proponga el docente dentro del medio virtual. Y la quinta dimensión es el ambiente de desarrollo virtual, es la diversa gama de estrategias utilizadas por el docente al evaluar a los estudiantes e interactuar asíncrona y sincrónicamente (Zambrano, 2016), Cabe mencionar, que es importante promover la comunicación, ya que permite interactuar con todos los estudiantes y entre ellos mismos, promoviendo un aprendizaje colaborativo.

\section{ESTRATEGIAS METODOLÓGICAS}

\section{Metodología}

El presente trabajo fue de tipo aplicado, al utilizar un marco teórico el cual fue establecido por información ya generada, que permitió actuar, modificar o alterar ámbitos donde se ha identificado el problema. Para Gabriel (2017), este tipo de investigación tiene como propósito aplicar la información obtenida en un ámbito determinado con el objetivo de solucionar dicho problema. Además, es de enfoque cuantitativo, ya que se optó por un procedimiento secuencial de medición para que luego, se realice una prueba de hipótesis por medio de análisis estadístico. Al respecto, Pinto (2018), mencionó que el enfoque cuantitativo, posee procedimientos de recolección y análisis de la información con el fin de responder a la pregunta de investigación sometiendo a prueba la hipótesis establecida con anterioridad, confiando en la cuantificación de la información utilizando estadística para evidenciar patrones conductuales de la muestra considerada.

De método hipotético deductivo, porque se formuló supuestos que se confrontaron con los datos recolectados de ambas variables de estudio determinando su veracidad o falsedad mediante métodos estadísticos. Al respecto, Pimienta y de la Orden (2017), dieron a entender que dicho método parte de enunciados generalizados o hallazgos de 
trabajos anteriores considerados para el presente trabajo, derivando hipótesis, corroboradas o rechazadas por medios estadísticos. Sobre nivel fue explicativo, ya que se identificó la causalidad, explicando de qué manera la educación virtual influye en la satisfacción escolar. Para Mendoza y Ramírez (2020), el nivel explicativo, tiene como propósito buscar la razón o causalidad generada por algún fenómeno, explicando el porqué de dicha ocurrencia, identificando y analizando las causalidades.

Finalmente, el diseño del presente trabajo fue el no experimental, por no haber manipulación alguna, y correlacional causal, ya que se pretende explicar el nivel de influencia de la educación virtual en la satisfacción escolar. Cabe señalar, que a continuación se muestra el esquema del presente trabajo el cual fue adaptado de Hernández et ál. (2014, p. 157).

$$
\mathrm{X}_{1} \rightarrow \mathrm{X}_{2}
$$

\section{Donde:}

X1 : Educación virtual (variable independiente)

X2 : Satisfacción escolar (variable dependiente)

$\rightarrow \quad$ : Influencia de la variable independiente sore la variable dependiente.

\section{Población y muestra}

Sobre la población la conformaron los 157 estudiantes pertenecientes a una institución educativa pública de nivel secundaria de Huancapi, Ayacucho. Para Hernández et ál. (2014), la población es una agrupación de elementos que tienen la particularidad de poseer caracteres similares de percepción, lugar o tiempo.

Tabla 1. Población

\begin{tabular}{cc}
\hline Grado y sección & $\mathbf{N}^{\circ}$ de Estudiantes \\
\hline $1^{\circ} \mathrm{A}$ & 18 \\
$1^{\circ} \mathrm{B}$ & 19 \\
$2^{\circ} \mathrm{A}$ & 16 \\
$2^{\circ} \mathrm{B}$ & 17 \\
$3^{\circ} \mathrm{A}$ & 12 \\
$3^{\circ} \mathrm{B}$ & 13 \\
$4^{\circ} \mathrm{A}$ & 15 \\
$4^{\circ} \mathrm{B}$ & 15 \\
$5^{\circ} \mathrm{A}$ & 16 \\
$5^{\circ} \mathrm{B}$ & 16 \\
\hline Total & 157 \\
\hline
\end{tabular}


Para definir a la muestra, se utilizó muestreo probabilístico estratificado, definiendo en primer lugar el tamaño muestral con apoyo de la fórmula finita, la cual evidenció que dicho tamaño es de 112 estudiantes, seguidamente se halló el factor proporcional para que la representatividad de cada grado y sección se mantenga, en tal sentido se ha dividido el tamaño muestral (112), con el tamaño poblacional (157), obteniéndose 0,713 de resultado que será multiplicado por la cantidad de estudiantes de cada grado y sección y de esa forma se obtendría la muestra.

Tabla 2. Muestra

\begin{tabular}{cccc}
\hline Grados y sección & Población & Factor & Muestra \\
\hline $1^{\circ} \mathrm{A}$ & 18 & 0.713 & 13 \\
$1^{\circ} \mathrm{B}$ & 19 & 0.713 & 14 \\
$2^{\circ} \mathrm{A}$ & 16 & 0.713 & 11 \\
$2^{\circ} \mathrm{B}$ & 17 & 0.713 & 12 \\
$3^{\circ} \mathrm{A}$ & 12 & 0.713 & 9 \\
$3^{\circ} \mathrm{B}$ & 13 & 0.713 & 9 \\
$4^{\circ} \mathrm{A}$ & 15 & 0.713 & 11 \\
$4^{\circ} \mathrm{B}$ & 15 & 0.713 & 11 \\
$5^{\circ} \mathrm{A}$ & 16 & 0.713 & 11 \\
$5^{\circ} \mathrm{B}$ & 16 & 0.713 & 11 \\
\hline Total & $\mathbf{1 5 7}$ & & $\mathbf{1 1 2}$ \\
\hline
\end{tabular}

\section{Técnicas e instrumentos}

La técnica seleccionada fue la encuesta para ambas variables. Al respecto, Gonzáles et ál. (2011), mencionaron que la encuesta es un proceso secuenciado basado en la interrogación sistematizada y planificada, la cual es aplicada a una cierta muestra seleccionada para llegar a conocer aspectos claves referente a algún tema en específico. Y como instrumentos se crearon dos cuestionarios, los cuales se caracterizan por su fácil aplicación y basta recolección de información por medio de ítems creados considerando a los indicadores detectados de las dimensiones que desglosan a la variable (Páramo, 2017).

Respecto a la validez de los instrumentos, se sometieron a la decisión de expertos, los cuales dieron su opinión concluyente, asimismo, la confiabilidad, se dio por medio del coeficiente de alfa de Cronbach, evidenciando que el cuestionario sobre educación virtual tuvo un índice de confiabilidad de 0,947 y el cuestionario sobre satisfacción escolar de 0,971 ; concluyendo que ambos instrumentos son confiables. 


\section{RESULTADOS Y DISCUSIÓN}

\section{Resultados descriptivos:}

Tabla 3. Tabla cruzada de educación virtual y satisfacción escolar

\begin{tabular}{|c|c|c|c|c|c|c|}
\hline & & & \multicolumn{3}{|c|}{ Satisfacción escolar } & \multirow[t]{2}{*}{ Total } \\
\hline & & & Bajo & Medio & Alto & \\
\hline \multirow{6}{*}{$\begin{array}{l}\text { Educación } \\
\text { virtual }\end{array}$} & \multirow{2}{*}{ Ineficiente } & Recuento & 2 & 0 & 1 & 3 \\
\hline & & $\%$ del total & $1,8 \%$ & $0,0 \%$ & $0,9 \%$ & $2,7 \%$ \\
\hline & \multirow{2}{*}{ Moderado } & Recuento & 1 & 27 & 9 & 37 \\
\hline & & $\%$ del total & $0,9 \%$ & $24,1 \%$ & $8,0 \%$ & $33,0 \%$ \\
\hline & \multirow{2}{*}{ Eficiente } & Recuento & 0 & 19 & 53 & 72 \\
\hline & & $\%$ del total & $0,0 \%$ & $17,0 \%$ & $47,3 \%$ & $64,3 \%$ \\
\hline \multirow{2}{*}{\multicolumn{2}{|c|}{ Total }} & Recuento & 3 & 46 & 63 & 112 \\
\hline & & $\%$ del total & $2,7 \%$ & $41,1 \%$ & $56,3 \%$ & $100,0 \%$ \\
\hline
\end{tabular}

Interpretación: De la tabla 3, se evidencia que del 100\% (112) de los estudiantes pertenecientes a la muestra el 2,7\% afirmó que la educación virtual se percibió en un nivel ineficiente, el $33 \%$ en nivel moderado y el 64,3\% en nivel eficiente, asimismo el 2,7\% de estudiantes señalo que la satisfacción escolar es baja, el 41,1\% se encuentra en nivel medio y el $56,3 \%$ en nivel alto.

\section{Resultados inferenciales}

Tabla 4. Informe de ajuste de modelo

\begin{tabular}{lrrrrr}
\hline \multicolumn{1}{c}{ Modelo } & Logaritmo de la verosimilitud -2 & $\begin{array}{c}\text { Chi- } \\
\text { cuadrado }\end{array}$ & gl & Sig. \\
\hline Sólo intersección & 51,328 & & & & \\
Final & 20,291 & 31,037 & 2 &, 000 \\
\hline
\end{tabular}

Función de enlace: Logit.

Interpretación: El grado de significancia fue de 0,000 menor a 0,05, por tanto, se puede afirmar que el modelo propuesto de educación virtual es apropiado, concluyendo que la educación virtual influye de manera significativa en la satisfacción escolar.

Tabla 5. Prueba Pseudo R cuadrado

\begin{tabular}{lr}
\hline Cox y Snell &, 242 \\
Nagelkerke &, 305 \\
McFadden &, 176 \\
\hline
\end{tabular}

Función de enlace: Logit. 
Interpretación: La prueba de Pseudo $\mathrm{R}^{2}$, permite deducir que, según Cox y Snell, el modelo de educación virtual explica a la variable satisfacción escolar en un 24,2\% y según Nagelkerke en un $30,5 \%$.

\section{Discusión}

Se llegó a determinar que el modelo propuesto de educación virtual es plausible, según el Pseudo $\mathrm{R}^{2}$ de Cox y Snell el modelo explica a la satisfacción escolar en 24,2\% y para Nagelkerke en 30,5\%, llegando a concluir que la educación virtual implementada apropiadamente provoca que la satisfacción escolar varie de forma significativa, tales hallazgos son análogos a los que obtuvo Valdez (2018), quien estableció la relación entre educación virtual y satisfacción estudiantil, estudio de tipo aplicado y de nivel correlacional, concluyendo que existe relación entre ambas variables con un Rho=0,757 $(0,000<0,05)$, los cuales fueron confirmados por los hallazgos descriptivos, los cuales mostraron que el 44,4\% de estudiantes mencionó que la educación virtual está en un nivel medio y el 34,3\% en nivel alto; respecto a la satisfacción estudiantil el 55,6\% mencionó que se encuentra en nivel alto. Asimismo, Núñez (2020), señaló que son alternativas de solución a la demanda educativa la instauración idónea de una educación virtual, ya que apoya a la formación de los estudiantes al incorporar herramientas tecnológicas novedosas y llamativas para el estudiante.

Al respecto, referente a la teoría constructivista, la cual en esencia menciona que las personas mediante su accionar físico y mental construye su propio conocimiento (Vega et ál., 2019), explica el hallazgo obtenido, ya que al utilizar entornos virtuales de aprendizaje los cuales apoyan a la instauración de la educación virtual, toman en cuenta al estudiante y le dan el protagonismo principal, arquitecto de su propio conocimiento. Finalmente, Baños et ál. (2017), mencionaron que, si el estudiante llega a sentir comodidad, gusto o satisfacción dentro de la escuela, dicha percepción contribuye a reducir los índices de abandono escolar y el comportamiento disruptivo.

Cabe señalar, que, en la actualidad, se ha implementado de manera forzosa la educación virtual, ya que se tiene la necesidad de brindar una educación de calidad y llevar la atención educativa a todo rincón del país, en tal sentido es lógico que se haya llegado a cometer errores en su implementación, y por ende, en algunos casos, ha surgido desconfianza e insatisfacción de parte de los estudiantes como de los padres de familia. 


\section{CONCLUSIÓN O CONSIDERACIONES FINALES}

El nivel de educación virtual percibida por los estudiantes de una institución educativa de Ayacucho evidencia que el 64,3\% (72) de los estudiantes coinciden que la educación virtual implementada es eficiente, asimismo respecto a la satisfacción escolar el 56,3\% (63), señalo que está en un nivel alto su satisfacción con las acciones tomadas por la institución educativa al brindarle una educación de calidad en la actualidad.

La educación virtual influye en la satisfacción escolar con un 24,2\% de incidencia según Cox y Snell y un 30,5\% según Nagelkerke, es decir, que si la educación virtual es implementada de manera apropiada provoca una variación significativa en la satisfacción escolar de los estudiantes de una institución educativa de Ayacucho.

\section{LISTA DE REFERENCIAS}

Ahmadi, J., y Nourabadi, S. (2020). Barreras de implementación en la educación virtual en la Universidad Payame Noor en Irán. Utopía y Praxis Lationoamericana, 25(2), 202-210. https://www.redalyc.org/journal/279/27963185021/html/

Baños, R., Ortíz-Camacho, M. M., Baena-Extremera, A., y Tristán-Rodríguez, J. L. (2017). Satisfaccion, motivación y rendimiento académico en estudiantes de Secundaria y Bachillerato: antecedentes, diseño, metodología y propuesta de análisis para un trabajo de investigación. Revista Digital del Centro del Profesorado Cuevas-Olula (Almería), 10(20), 40-50. https://dialnet.unirioja.es/servlet/articulo?codigo=5900741

Cáceres, Z., y Munévar, O. (2016). Evolución de las teorías cognitivas y sus aportes a la educación. Revista Actividad Física y Desarrollo humano, 7(1), 1-13. https://doi.org/10.24054/16927427.v2.n2.2016.2408

Crisol-Moya, E., Herrera-Nieves, L. y Montes-Soldado, R. (2020). Educación virtual para todos: una revisión sistemática. Education in the knowledge Society, 21(15), 1-13. https://www.researchgate.net/publication/342907961_Educacion_virtual_para_t odos_una_revision_sistematica

De Luca, M. P. (2020). Las aulas virtuales en la formación docente como estrategia de continuidad pedagógica en tiempos de pandemia, Usos y paradojas. Análisis Carolina, Serie: formación virtual. 33(1), 1-12. https://dialnet.unirioja.es/descarga/articulo/7439302.pdf 
Esteve, F., Castañada, L., y Adell, J. (2018). Un modelo holístico de competencia docente para el mundo digital. Revista interuniversitaria de formación del profesorado, 91(32), 105-116. https://dialnet.unirioja.es/servlet/articulo?codigo=6441415

Gabriel, J. (2017). Cómo se genera una investigación científica que luego sea motivo de publicación. Journal of the Selva Andina Research Society, 8(2), 155-156. http://www.scielo.org.bo/scielo.php?script=sci_arttext\&pid=S207292942017000200008

Gonzales, A., Oseda, D., Ramírez, F., y Gave, J. (2011). ¿Cómo aprender investigación científica? (1ra Edición). Huancavelica.

González-Peitado, M., Pino-Juste, M., y Penado, M. (2017). Estudio de la satisfacción percibida por los estudiantes de la UNED con su vida universitaria. RIED. Revista Iberoamericana de Educación a Distancia, 20(1), 243-260. https://www.redalyc.org/pdf/3314/331450972013.pdf

Gros, B. (2011). Evolución y retos de la Educación virtual. Construyendo el E-Learning del siglo XXI. Editorial UOC. https://redined.mecd.gob.es/xmlui/bitstream/handle/11162/66735/008201220165 79.pdf? sequence $=1 \&$ is Allowed $=\mathrm{y}$

Gros, B. (2018). La evolución del e-learning: del aula virtual a la red. Revista Iberoamericana de Educación a Distancia, 21(2), 69-82. https://doi.org/10.5944/ried.21.2.20577

Hernández, R., Fernández, C. y Baptista, P. (2014). Metodología de la investigación (6ta. Edición). McGraw-Hill. https://www.uca.ac.cr/wpcontent/uploads/2017/10/Investigacion.pdf

Manrique, K. A., y Sánchez M. (2018). Satisfacción estudiantil universitaria: un referente para elevar los indicadores de los cursos en línea impulsados por la Coordinación General de Educación Virtual de la UAGro. Cuaderno de Pedagogía Universitaria, $\quad 16(31)$, 17-30. https://dialnet.unirioja.es/servlet/articulo?codigo $=6855124$

Martínez, J. E., Segobia, M. A., y Alipio, J. (2019). Tecnología: La educación virtual y su aporte al desarrollo humano. Revista dilemas Contemporáneos: Educación, $\begin{array}{llll}\text { Política } & y & \text { Valores, } & \text { 7(19), }\end{array}$ 
http://search.ebscohost.com/login.aspx?direct=true \&db=eue\&AN=139571989\& lang $=$ es\&site $=$ eds-live

Mendoza, M. L., y Rodríguez M. (2019). Aprendizaje centrado en el estudiante desde la planificación en investigación. CIENCIAMATRÍA, 6(10), 560-572. https://doi.org/10.35381/cm.v6i10.232

MINEDU (2020). R.M. N¹60-2020-MINEDU. Disponen el inicio del año escolar a través de la implementación de la estrategia denominada "Aprendo en casa", a partir del 6 de abril de 2020 y aprueban otras disposiciones. https://www.gob.pe/institucion/minedu/normas-legales/466108-160-2020minedu

Ministerio de Educación de Argentina (2020). Boletín Especial de la República de Argentina. Legislación y avisos Oficiales. Resolución 104/2020. https://www.boletinoficial.gob.ar/\#!DetalleNorma/226749/20200316

Morán, F. J., Rosero, J. M., y Olvera, L. A. (2017). Recursos tecnológicos. Grupo Compás.

http://142.93.18.15:8080/jspui/bitstream/123456789/140/1/LIBRO\%20RECUR SOS\%20TECNOLOGICOS-ilovepdf-compressed.pdf

Naciones Unidas (2020). Informe de políticas: La educación durante la COVID-19 y después de ella. https://www.un.org/sites/un2.un.org/files/policy_brief__education_during_covid-19_and_beyond_spanish.pdf

Núñez, V. E. (2020). Impacto de la educación virtual en el deporte en la república de Colombia. Revista $\quad$ Edu-Física, $12(25), \quad$ 131-139. http://revistas.ut.edu.co/index.php/edufisica/article/view/2085

Páramo, P. (2017). La investigación en ciencias sociales: Técnicas de recolección de información. (1ra. Edición). Universidad Piloto de Colombia.

Peche, H. J., y Giraldo, V. E. (2019). El aprendizaje Flip Learning centrado en el estudiante como generador de calidad educativa. Revista Arbitrada Interdisciplinaria $\quad$ KPINONIA, 4(4), 427-450. http://dx.doi.org/10.35381/r.k.v4i8.293

Pimienta, J. G. y de la Orden, A. (2017). Metodología de la investigación. (3ra. Edición). Pearson Educación México, de S.A. http://repositorio.uasb.edu.bo:8080/bitstream/54000/1268/1/Pimienta- 
Metodolog\%C3\%ADa\%20de\%201a\%20investigaci\%C3\%B3n\%203ra\%20ed.pd f

Pinto, J. E. M. (2018). Metodología de la Investigación social: Paradigmas: cuantitativo, sociocrítico, cualitativo, complementario. (1ra. Edición). Ediciones de la U.

Tomás, J.-M., y Gutiérrez, M. (2019). Aportaciones de la teoría de la autodeterminación a la predicción de la satisfacción escolar en estudiantes universitarios. Revista De Investigación Educativa, 37(2), 471-485. https://doi.org/10.6018/rie.37.2.328191

UNESCO (2020). Informe COVID-19, CEPAL-UNESCO. La educación en tiempos de la pandemia de COVID-19. https://unesdoc.unesco.org/ark:/48223/pf0000374075?locale=es

Valdez, E. B. (2018). La educación virtual y la satisfacción del estudiante en los cursos virtuales del Instituto Nacional Materno Perinatal 2017 [Tesis de maestría, Universidad César Vallejo]. https://hdl.handle.net/20.500.12692/21504

Vega-Lugo, N., Flores-Jiménez, R., Flores-Jiménez, I., Hurtado-Vega, B., y RodríguezMartínez, J. S. (2019). Teoría del aprendizaje. XIKUA Boletín Científico de la Escuela Superior de Tiahuelilpan, 7(14), 51-53. https://repository.uaeh.edu.mx/revistas/index.php/xikua/article/view/4359/6343

Zambrano, J. (2016). Factores predictores de la satisfacción de estudiantes de cursos virtuales. RIED Revista Iberoamericana de Educación a Distancia, 19(2), 217235. http://revistas.uned.es/index.php/ried/article/view/15112 